\title{
Androgen receptor induces EPHA3 expression by interacting with transcription factor SP1
}

\author{
XIAOWEI DIAO ${ }^{1}$, XIUBIN CHEN $^{1}$, YURUI PI $^{1}$, YU ZHANG $^{1}$, FANGFANG WANG $^{1}$, PING LIU $^{1}$, \\ YANHONG GAO $^{1}$, XIAOJUN WANG ${ }^{1}$, SIJUN YANG ${ }^{2}$ and SHAN LU ${ }^{1}$
}

\begin{abstract}
${ }^{1}$ School of Life Sciences, Jiangsu Key Laboratory for Molecular and Medical Biotechnology, Nanjing Normal University, Nanjing, Jiangsu 210023; ${ }^{2}$ ABSL-III Laboratory, Center for Animal Experiments and State Key Laboratory of Virology, Wuhan University School of Medicine, Wuhan, Hubei 430071, P.R. China
\end{abstract}

Received December 5, 2017; Accepted June 8, 2018

DOI: $10.3892 /$ or.2018.6503

\begin{abstract}
Erythropoietin-producing hepatocellular carcinoma cell surface type-A receptor 3 (EPHA3) has been found to promote the proliferation and survival of prostate cancer (PCa) cell lines and prostate tumor development in nude mice. However, the regulation of EPHA3 in PCa remains largely unknown. This study is aimed to investigate the association between EPHA3 expression and androgen receptor (AR) signaling and the potential mechanism. We determined mRNA and protein levels of EPHA3 and AR signaling-related genes in the PCa cell line 22Rv1 by reverse transcription-polymerase chain reaction (RT-PCR) and western blotting, respectively. The EPHA3 mRNA and protein levels were both found to be elevated by dihydrotestosterone (DHT) hormone in a doseand time-dependent manner, as AR and prostate-specific antigen (PSA) expression were increased. Similarly, EPHA3 protein levels were also increased in the PCa cell line LNCaP stimulated with DHT or mibolerone (Mib). Overexpression of
\end{abstract}

Correspondence to: Dr Shan Lu, School of Life Sciences, Jiangsu Key Laboratory for Molecular and Medical Biotechnology, Nanjing Normal University, 1 Wenyuan Road, Qixia, Nanjing, Jiangsu 210023, P.R. China

E-mail: lu_shan@hotmail.com

Abbreviations: EPHA3, erythropoietin-producing hepatocellular carcinoma cell surface type-A receptor $3 ; \mathrm{PCa}$, prostate cancer; $\mathrm{AR}$, androgen receptor; RT-PCR, reverse transcription-polymerase chain reaction; qPCR, quantitative fluorescence real-time PCR; PSA, prostate-specific antigen; ARE, androgen response element; Sp, specific protein; siRNA, small interfering RNA; Co-IP, co-immunoprecipitation; ChIP, chromatin IP; RMS, rhabdomyosarcoma; FBS, fetal bovine serum; SFBS, charcoal/dextran-stripped FBS; DHT, dihydrotestosterone; Mib, mibolerone; MTM, mithramycin A; PMSF, phenylmethylsulfonyl fluoride; LBD, ligand binding domain; VEGF, vascular endothelial growth factor

Key words: EphA3, androgen receptor, SP1, prostate cancer, transcription activity, regulation
pEGFP-AR in 22Rv1 cells significantly increased the EphA3 level, while AR knockdown with small interfering RNA (siRNA) for AR (siAR) markedly decreased the expression of EPHA3. The key EPHA3 promoter region associated with AR regulation was evaluated by co-transfection of various pGL3-basic-luciferase reporter plasmids, containing EPHA3 core promoter fragments differing in length, with the AR plasmid or siAR into $22 \mathrm{Rv} 1$ cells. AR overexpression in 22Rvl cells raised the EphA3 promoter transcription activity of pGL3-EPHA3-Luc (EPHA3-Luc)-789, and vice versa. Similarly, luciferase activity of EPHA3-Luc-317 was also clearly affected. However, truncated EPHA3-Luc-237 without the transcription factor specific protein 1 (SP1) binding sites or EPHA3-Luc-789 $\triangle$ SP1 with modified SP1 binding sites clearly decreased EPHA3 promoter activity regardless of whether AR was overexpressed or blocked. Treatment of 22Rv1 cells with 10 and $100 \mathrm{nM}$ of the SP1 inhibitor mithramycin A for 24 and $48 \mathrm{~h}$ significantly reduced EPHA 3 mRNA and protein levels. Additionally, selective inhibition of SP1 with siRNA SP1 (siSP1) at various concentration from 25 to $75 \mathrm{nM}$, reduced the EPHA3 protein level in PCa LNCaP cells, accordingly. Co-immunoprecipitation (co-IP) and chromatin IP (ChIP) assays were performed to determine whether AR forms a transcription factor complex with $\mathrm{Sp} 1$ that binds the EPHA3 core promoter region to sense androgen induction. The result suggests that the interaction of AR and SP1 contributes to regulate EPHA3 expression, and the SP1 binding sites (-295 -261) in the EPHA3 core promoter region is crucial to the regulation of EPHA3 expression in response to androgen hormone stimuli.

\section{Introduction}

Erythropoietin-producing hepatocellular carcinoma cell surface type-A receptor 3 (EPHA3) is a member of the Eph subfamily of receptor tyrosine kinases, and is structurally classified into cytoplasmic and extracellular regions. The cytoplasmic region is composed of a regulatory juxtamembrane domain with two tyrosine residues, a tyrosine kinase domain, a sterile- $\alpha$-motif interaction domain and a PSD95/Dlg/ZO-1 binding motif. The extracellular region contains a cysteine-rich domain comprised of a sushi and epidermal growth factor-like domain, two fibronectin III repeats, and an N-terminal 
globular ligand-binding domain (LBD) that can interact with cell membrane-bound ligands. These ligands mainly include ephrin A3, ephrinA5, or ephrinB2, which trigger bidirectional signaling of tyrosine kinase-dependent and independent pathways involved in cell communication. EPHA3 is highly expressed during the embryonic development of the brain and spinal cord, lungs, kidneys and heart, and then drops to a low level in most normal adult tissues (1). However, its expression is also elevated in a wide range of malignancies, including gastric cancer (2,3), melanoma (4-6), hepatocellular carcinoma (7) and glioblastoma (8), and is correlated with tumorigenicity, tumor angiogenesis and cancer progression (7-9). It was found that blocking the activation of the EPHA3 receptor with soluble EPHA3-Fc inhibited tumor growth in the 4T1 model of metastatic mammary adenocarcinoma (10). The first-in-class monoclonal antibody KB004 targeting the overexpressed receptor tyrosine kinase EPHA3 was shown to be clinically active against refractory hematological malignancies in humans (11). The conjugate cytotoxin of ephrin A5-Fc and PE38QQR potently and specifically killed glioblastoma tumor cells (12). The EPHA3-specific monoclonal antibody IIIA4 was also found to have antitumor effects in EPHA3-expressing leukemic xenografts (13). Accordingly, EPHA3 has been paid more attention as one of the promising targets for the treatment of several cancers $(14,15)$. Conversely, there are also contradictory reports concerning EphA 3 expression in tumors and its effect on the regulation of cancer progression. EPHA3 expression is more commonly downregulated and does not play a major role in colorectal cancer (16-18). EPHA3 is not highly expressed in the primary tumor but in lymph node metastases, and its expression also varies with disease stage in breast cancer (19). EPHA3 was found to suppress cell adhesion and migration when EPHA3 phosphorylation was increased by ephrinA5 stimulation in EPHA3-expressing TE671 and RD rhabdomyosarcoma (RMS) cells, or when EPHA3 was ectopically expressed in the EPHA3-negative CRL2061 RMS cell line (20), but EPHA3 and ephrinA1 upregulated by the transcription factor PAX7 in RMS cell lines was associated with cell migration and invasiveness (21). In addition, EPHA3 overexpression in human lung cancer cell lines increased apoptosis by suppressing AKT activation in vitro and inhibited the growth of tumor xenografts (22). It also decreased chemoresistance of small cell lung cancer via the PI3K/BMX/STAT3 signaling pathway (23). Furthermore, the EphA3 level was found to be lower in primary lung adenocarcinomas than in normal clinical specimens (22). Given the above-mentioned findings, the role of EPHA3 either in promoting or suppressing oncogenesis is quite complex and paradoxical in a variety of cancers.

Noteworthy, production of higher amounts of EPHA3 is reported in the normal prostate compared with other benign human tissues, such as in the uterus and bladder (24). However, although EPHA3 is also found to be expressed in prostate tumor vasculature and stroma, there is a great disparity in the EPHA3 levels, either elevated or reduced, in various prostate cancer (PCa) cell lines compared with normal prostate epithelium cells $(25,26)$. In addition, a positive correlation between the levels of EPHA3 and the Gleason grade of PCa has also been found in clinical PCa specimens (26). In addition, EphA3 enhanced the proliferation and survival of PCa LNCaP cells and tumor development in nude mice subcutaneously implanted with EPHA3-overexpressing LNCaP cells, and EPHA3 inhibition suppressed the survival of the LNCaP-derived subline, C4-2B cells (27). The treatment of mice with an agonistic $\alpha$-EPHA3 antibody was found to inhibit tumor growth by activation of EPHA3(+)/CD90(+)/Sca1(+) mesenchymal/stromal cells (25). All of these findings highlight the importance of further investigating EPHA3 expression and the related mechanism in PCa. PCa is the second most common carcinoma and the fifth leading cause of cancer-related deaths in men worldwide (28), in which the androgen receptor (AR) plays a pivotal role in the initiation and progression of PCa (29-31). The regulation of EPHA3 in PCa remains mostly unclear, and particularly the association between AR and EPHA3 regulation has been less studied. In the present study, we aimed to reveal the effects of AR signaling on EPHA3 expression and the molecular mechanism of AR-regulated EPHA3 expression in androgen-dependent PCa cells, and identified the DNA binding sites within the EPHA3 promoter region that contribute to the regulation of the expression of EPHA3 in response to androgen hormone.

\section{Materials and methods}

Cell culture and chemical treatments. The media, fetal bovine serums (FBS) and charcoal/dextran stripped FBS (SFBS) for cell culture were all purchased from Gibco (Thermo Fisher Scientific, Inc., Waltham, MA, USA). Human prostate adenocarcinoma cell lines PC-3, DU145, LNCaP and 22Rv1 were obtained from the American Type Culture Collection (ATCC; Manassas, VA, USA) and maintained in RPMI-1640 medium containing $10 \% \mathrm{FBS}, 100 \mathrm{IU} / \mathrm{ml}$ penicillin and $0.1 \mathrm{mg} / \mathrm{ml}$ streptomycin at $37^{\circ} \mathrm{C}$ in $5 \% \mathrm{CO}_{2}$. Human immortalized prostatic myofibroblast stromal cell line WPMY-1 was obtained from Xiangf Bio Technology Co., Ltd. (Shanghai, China) and maintained in Dulbecco's modified Eagle's medium (DMEM) with 10\% FBS. The androgens DHT and Mib were obtained from Toronto Research Chemicals (North York, ON, Canada) and J\&K Scientific (Beijing, China), respectively, and then dissolved in ethanol at a stock concentration of $10^{-2}$ mol/1. Mithramycin A (MTM) was obtained from Bio Basic (Markham, ON, Canada) and dissolved in dimethyl sulfoxide (DMSO) to prepare a $1 \mathrm{mM}$ stock solution. For androgen stimulation, cells maintained in phenol-free media with $10 \%$ FBS for 3-5 days were plated in 6-well dishes and allowed to grow to near confluence. Subsequently, the confluent cells were treated with androgen at different concentrations for various time periods as noted in the Figure legends. For MTM treatment, cells close to confluence were treated in detail as noted in the Figure legends.

Reverse transcription-polymerase chain reaction (RT-PCR) and quantitative fluorescence real-time PCR (qPCR). Total RNA from cells was prepared with TRIzol ${ }^{\circledR}$ reagent (Thermo Fisher Scientific, Inc.) according to the manufacturer's instructions. RT reaction was performed with the HiScript Q RT SuperMix, PCR was carried out with a 2X Taq Master Mix, and real-time monitoring PCR was conducted with the AceQ qPCR SYBR-Green Master Mix (all from Vazyme Biotech, Nanjing, China) using the Applied Biosystems StepOnePlus 
Table I. Primers used for the construction of luciferase reporter plasmids.

Name

Primers $^{\mathrm{a}}$

-789-EphA3-P-F

-789/-317/-237-EphA3-P-R

-317-EphA3-P-F

-237-EphA3-P-F

$\Delta \mathrm{Sp} 1-\mathrm{F}$

$\Delta \mathrm{Sp} 1-\mathrm{R}$

\author{
CCGCTCGAGCTCCCTCCGTAAGATGA \\ CCCAAGCTTCTTTGAGAGCGTGAGCC \\ CCGCTCGAGCTTCTTGTTTCCTGTGG \\ CCGCTCGAGTGCCATCCCGCTCTGCTT
}

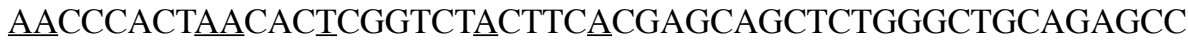

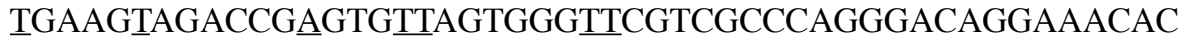

${ }^{\mathrm{a}}$ Mutation sites are underlined.

Real-Time PCR System (Thermo Fisher Scientific, Inc.). Primers specific for AR (32), PSA (33), EPHA3 (2), SP1 (34) and $\beta$-actin (forward, 5'-GAGCTACGAGCTGCCTGACG-3' and reverse, 5'-CCTAGAAGCATTTGCGGTGG-3') were synthesized by Shanghai Generay Biotech Co., Ltd. (Shanghai, China). Reaction mixtures were applied to gel electrophoresis immediately after PCR amplification. In the RT-PCR analysis, the expression of the target genes was normalized to $\beta$-actin levels in the respective samples as an internal control, and in the $\mathrm{qPCR}$, the cycle threshold values were used to calculate the normalized expression of the target genes against $\beta$-actin using the Q-Gene software (http://www.gene-quantification. de/download.html\#qgene) (35).

Antibodies and western blotting. Antibodies against AR (dilution 1:1,000; cat. no. sc-816), EPHA3 (dilution 1:1,000; cat. no. sc-919), or SP1 (dilution 1:1,000; cat. no. sc-14027) and the horseradish peroxidase-conjugated secondary antibodies for rabbit (dilution 1:5,000; cat. no. sc-2357) was purchased from Santa Cruz Biotechnology, Inc. (Santa Cruz, CA, USA). Antibody against PSA (dilution 1:2,000; cat. no. DAK-A056201) was obtained from Dako (Glostrup, Denmark) and against $\beta$-actin (dilution 1:10,000; cat. no. AP0060) was obtained from Bioworld Technology (St. Louis Park, MN, USA). The cells were lysed in ice-cold RIPA buffer (Beyotime Institute of Biotechnology, Haimen, China) supplemented with $1 \mathrm{mM}$ phenylmethylsulfonyl fluoride (PMSF; Sigma-Aldrich; Merck KGaA, Darmstadt, Germany) and protease inhibitor cocktail (Roche Applied Science; Roche Diagnostics Corporation, Indianapolis, IN, USA) for quantification with the DC Protein Assay kit purchased from Bio-Rad Laboratories (Hercules, CA, USA). Equal amounts of whole cell extracts were boiled for $5 \mathrm{~min}$ in sample loading buffer, separated by SDS-PAGE and transferred onto polyvinylidene difluoride membranes, which were probed with the indicated primary antibody followed by incubation with the appropriate secondary antibody. After washing, proteins were detected by a chemiluminescent detection system (Tanon 5200; Tanon Science and Technology, Co., Ltd., Shanghai, China). The software of ImageJ 1.42 (NIH, Bethesda, NY, USA) was used to perform densitometry analysis for protein semi-quantification.

Construction of plasmids and transient transfection of luciferase reporter and small interfering RNA (siRNA).
The firefly basic luciferase reporter vector pGL3-Basic and pEGFP-N1 plasmid were kindly provided by Dr Chuanjun Wen (Nanjing Normal University, Nanjing, China). The EPHA3 core promoter region (36) was analyzed using GeneCards (Weizmann Institute of Science, Rehovot, Israel) and the TRANSFAC databases (http://gene-regulation. com/, Gottingen, Germany) to search transcription factor binding sites for AR and SP1. All the primers designed for the construction of the reporter plasmids are summarized in Table I. The EPHA3-Luc-789 plasmid was generated by inserting a core promoter sequence of the EPHA3 gene spanning from -789 to +146 bp into the pGL3-Basic luciferase reporter vector. Construct EPHA3-Luc-317 is composed of a PCR fragment spanning from -317 to +146 bp of the EPHA3 promoter, and EPHA3-Luc-237 has core promoter sequence from -237 to +146 bp instead. The mutant EPHA3-Luc-789 $\Delta$ Sp1 was constructed mostly in the same way as EPHA3-Luc-789 except that the small fragment of the SP1 binding region was replaced by multi-point mutations using PCR amplification with the combinations of specifically designed longer oligonucleotide $\Delta \mathrm{Sp} 1-\mathrm{F}$ and the reverse primer for the EPHA3-Luc-789 construct, and the forward primer for EPHA3-Luc-789 and $\triangle$ SP1-R. The AR expression plasmid (pEGFP-AR) was constructed by inserting the full-length cDNA of the AR from LNCaP cells amplified by PCR using the forward primer attached with an EcoRI site (5'-CCGGAATTCATGGAAGTGCAGTTA GGGCTG-3') and the reverse primer with a BamHI site (5'-CGCGGATCCTCACTGGGTGCGCGGATCCTCACT GGGTGTGGAAATAGATGG-3') added into the multiple cloning site of pEGFP-N1. PCa cells in 24-well plates were transiently transfected with $0.4 \mu \mathrm{g}$ of the indicated reporter plasmid, $0.4 \mu \mathrm{g}$ of pEGFP-AR or $50 \mathrm{nM}$ of siRNA AR (siAR) (37), and $0.01 \mu \mathrm{g}$ pRL-TK as an internal control using Lipofectamine 2000 transfection reagent (Thermo Fisher Scientific, Inc.). After $48 \mathrm{~h}$ transfection, cell lysates were prepared according to the Dual-Luciferase Reporter Assay system (Promega Corporation, Madison, WI, USA) protocol for the luciferase assay in a Luminoskan Ascent (Thermo Fisher Scientific, Inc.), and firefly luciferase activities were corrected by the corresponding Renilla luciferase activities. The siAR and siRNA SP1 (siSp1) (20) were synthesized separately by Thermo Fisher Scientific, Inc., and transfected as indicated in the Figure legends. 
Co-IP and ChIP assay. The 22Rv1 cells were maintained in SFBS medium for 3-5 days and grown to confluence in $100-\mathrm{mm}$ dishes. Then, the cells were lysed in a modified RIPA buffer [50 mM Tris- $\mathrm{HCl}$ (pH 7.4), 1\% NP-40, $150 \mathrm{mM} \mathrm{NaCl}]$ containing $1 \mathrm{mM}$ PMSF and $1 \mathrm{X}$ protease inhibitor cocktail after stimulation with $10 \mathrm{nM}$ Mib for $24 \mathrm{~h}$, and centrifuged at $13,523 \mathrm{x} \mathrm{g}$, and $4^{\circ} \mathrm{C}$ for $15 \mathrm{~min}$. One aliquot of the supernatant was saved as the input control, the remainder was incubated with $1.5 \mu \mathrm{g}$ of anti-AR antibody, anti-SP1 antibody and negative control IgG antibody (cat. no. A7016; Beyotime Institute of Biotechnology), separately, followed by pull-down with $15 \mu \mathrm{l}$ Protein A/G PLUS-Agarose beads (Santa Cruz Biotechnology, Inc.). The beads were then collected by centrifugation at $845 \mathrm{x}$ g for $5 \mathrm{~min}$ and washed three times with cold PBS. The immunoprecipitates were eluted by boiling in reducing sample buffer for 5 min and subjected to western blot analysis along with input sample as described above.

Concurrently, $8 \times 10^{6}$ cells starved in phenol-free RPMI-1640 medium with 10\% SFBS medium were stimulated with $10 \mathrm{nM}$ DHT for $24 \mathrm{~h}$, cross-linked by adding formaldehyde up to a final concentration of $1 \%$ at room temperature for $10 \mathrm{~min}$ and mixed with $0.125 \mathrm{M}$ glycine for $5 \mathrm{~min}$ to quench cross-linking. The cultures were then harvested by centrifugation and the pellets were washed twice with ice-cold PBS and lysed in $300 \mu \mathrm{l}$ of lysis buffer [50 mM Tris- $\mathrm{HCl}$ (pH 8.0), $10 \mathrm{mM}$ EDTA and 1\% SDS] for shearing chromatin on ice in $5 \mathrm{sec}$ on $/ 15 \mathrm{sec}$ off pulses at $10 \%$ output for $10 \mathrm{~min}$ in a sonicator (Ningbo Scientz Biotechnology Co., Ltd., Ningbo, China). Subsequenlty, the lysates were centrifuged to separate cell debris from the supernatant, of which one tenth of the volume was saved as input sample and the remainder was diluted 10-fold with ChIP dilution buffer [16.7 mM Tris- $\mathrm{HCl}$ (pH 8.1), 1.2 mM EDTA, 167 mM NaCl, 1.1\% Triton X-100 and $0.01 \% \mathrm{SDS}$ ] including protease inhibitor. Subsequently, $500 \mu \mathrm{l}$ aliquots were incubated with $2 \mu \mathrm{g}$ of the appropriate antibodies or IgG as negative control at $4^{\circ} \mathrm{C}$ overnight, and $15 \mu 1$ Protein A/G PLUS-Agarose beads (cat. no. sc-2003; Santa Cruz Biotechnology, Inc.) were added for another 2-h incubation at room temperature with gentle rotation to immunoprecipitate protein-bound chromatin fragments. The beads were collected and washed orderly with low salt, high salt and $\mathrm{LiCl}$ buffers following the protocol of the ChIP assay kit (Upstate Biotechnology Inc., Lake Placid, NY, USA), and eluted in $100 \mu \mathrm{l}$ TE buffer $(20 \mathrm{mM}$ Tris- $\mathrm{HCl} \mathrm{pH} 8.0,1 \mathrm{mM}$ EDTA pH 8.0). Cross-linking for both the eluate and the input samples were reversed by incubation in $0.2 \mathrm{M} \mathrm{NaCl}$ at $65^{\circ} \mathrm{C}$ for $2 \mathrm{~h}$, and qPCR analysis was performed as described above. The primer pair for the EPHA3 promoter surrounding the SP1 binding sites is FW1 (5'-CACTCCCACCCGGTCTCCTT-3') and RV1 (5'-GGGTCCTAGTGCCGTCTT-3').

Image quantification and statistical analysis. The results are representative of at least three independent experiments. Images of the agarose gel electrophoresis and western blotting membranes were analyzed with Quantity One Software (Bio-Rad Laboratories) to quantify the mRNA and protein expression. The data are expressed as the mean with standard deviations for all groups, which were calculated using the GraphPad Prism 5 (GraphPad Software, Inc., La Jolla, CA, USA). Comparisons between two groups were performed using Student's t-test, and comparisons among multiple groups were conducted by one-way analysis of variance (ANOVA) using Bonferroni's post hoc test. Differences were considered to be statistically significant if P-value was $<0.05$.

\section{Results}

Androgen induces $m R N A$ and protein expression of EphA3 in PCa 22Rvl cells in a dose-and time-dependent manner. To determine whether EPHA3 expression is induced by androgen in PCa cells, androgen responsive 22Rv1 cells were stimulated with various doses of DHT for different time periods for RT-PCR and western blot analysis. The data presented in Fig. 1A reveals that with the increase of the DHT concentration, EPHA3, AR and PSA mRNA levels were gradually increased compared with the untreated control. Similarly, EPHA3, AR and PSA protein levels were also induced by DHT, as displayed in Fig. 1B, where the two bands for the AR protein in 22Rv1 cells correspond to one full-length and the other to a truncated AR lacking the $\mathrm{COOH}$-terminal domain (CTD). The mRNA and protein expression of EPHA3 was continually increased in a dose-dependent manner by treatment with DHT from 0.1 to $10 \mathrm{nM}$, in which EPHA3 expression was significantly increased at $1 \mathrm{nM}$ and achieved a more than 2 -fold increase at $5 \mathrm{nM}$ (Fig. 1A and B, right pattern). Similar time-dependent trends were observed for mRNA and protein expression of EPHA3, AR and PSA in 22Rv1 cells treated with $10 \mathrm{nM}$ DHT from time 0 to $72 \mathrm{~h}$ (Fig. 1C and D). In particular, EphA3 expression was significantly elevated after $8 \mathrm{~h}$ of DHT treatment, and up to more than 2-fold after $24 \mathrm{~h}$ of DHT treatment (Fig. 1C and D, right pattern). In addition, another androgen responsive $\mathrm{PCa}$ cell line, $\mathrm{LNCaP}$, was also stimulated by both DHT and Mib at different concentrations (Fig. 2), where it is shown that the EPHA3 protein level was gradually elevated along with the increases in AR and PSA levels.

$A R$ expression level affects EPHA3 $\mathrm{mRNA}$ and protein expression in PCa 22Rvl cells. To investigate the association between AR and EPHA3 expression, qPCR analysis and western blotting were performed to measure AR and EPHA3 expression in the prostate stromal cell WPMY-1 and PCa cell lines LNCaP, 22Rv1, PC-3 and DU145. The results shown in Fig. 3A and $\mathrm{B}$ revealed that the mRNA levels of EPHA3 and AR were higher in LNCaP and 22Rv1 cells, and almost undetectable in WPMY-1, PC-3, while DU145 cells hardly expressed AR, which was confirmed at the protein level, as displayed in Fig. 3C. To evaluate whether AR can regulate EPHA3 expression, AR was overexpressed or silenced to determine EPHA3 and PSA levels in 22Rv1 cells. The results showed that overexpression of AR markedly increased PSA and EPHA3 expression both at the mRNA (Fig. 3D) and protein levels (Fig. 3E). In contrast, AR-specific inhibition of siAR markedly decreased both the mRNA and protein expression of PSA and EPHA3 (Fig. 3D and E).

SP1 binding sites are required to regulate EPHA3 expression in response to AR signaling. To further investigate whether AR binds the EPHA3 promoter region and directly regulates EPHA3 transcription, two important transcription 
A

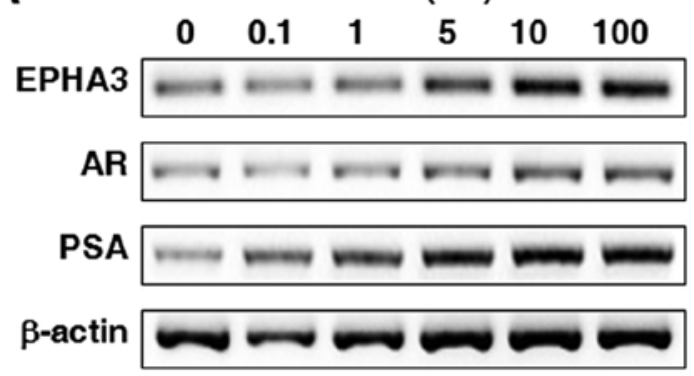

B

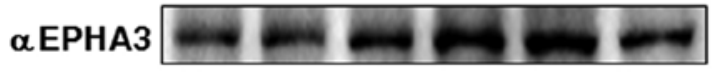

$\alpha$ AR

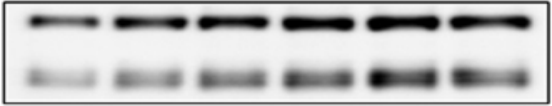

$\alpha$ PSA 2030

$\alpha \beta$-actin $=0$

22Rv1

C

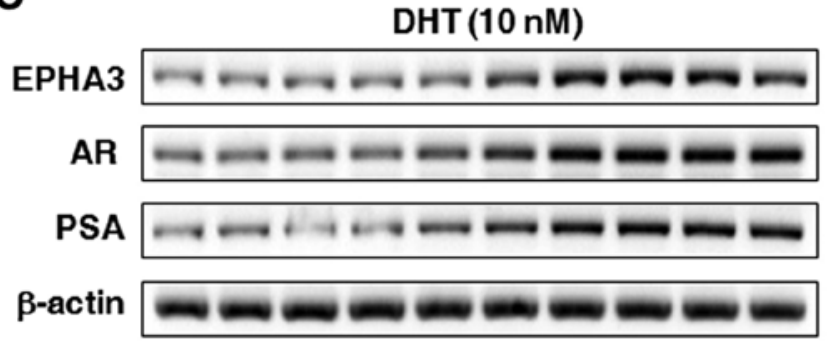

D

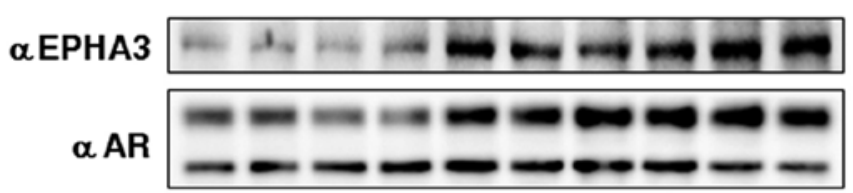

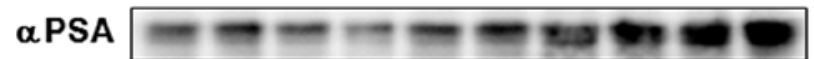

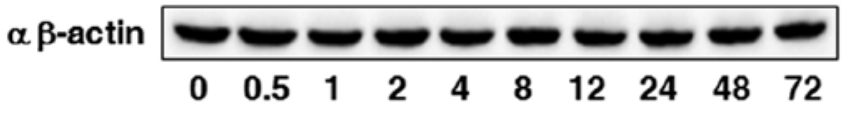

Time (h)
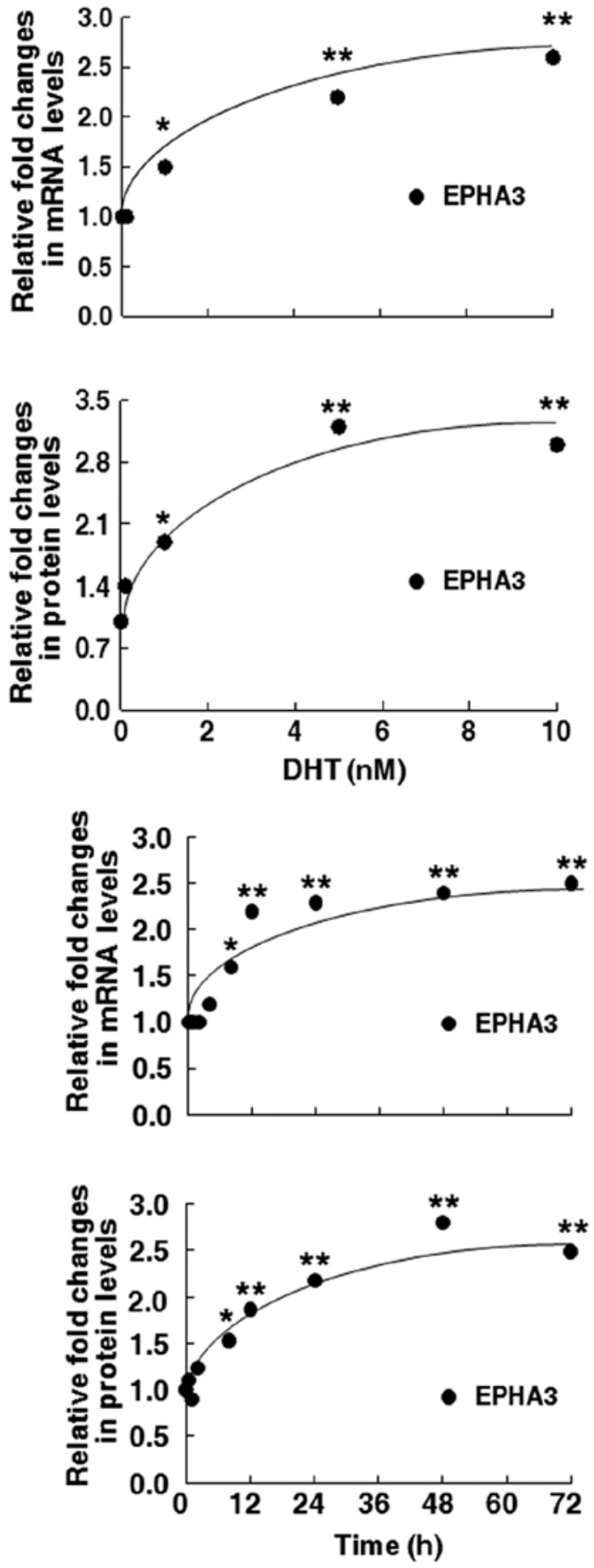

Figure 1. Dose-response and time course effect of DHT treatment on EPHA3 expression in prostate cancer 22Rv1 cells. (A and B) Following androgen starvation, 22Rv1 cells were treated with DHT at final concentrations of $0.1,1,5,10$ and $100 \mathrm{nM}$ for $24 \mathrm{~h}$, or (C and D) treated with $10 \mathrm{nM}$ DHT for 0-72 h. (A and C) RT-PCR or (B and D) western blotting was performed to measure mRNA and protein expression, respectively, for AR, PSA and EPHA3 levels as described in the text. $\beta$-actin levels were used as loading controls, and images of the agarose gel electrophoresis and western blotting membranes were analyzed by densitometry for quantification of the mRNA and protein expressions of AR, PSA and EPHA3, and the results are shown as relative fold-change, normalized to $\beta$-actin and relative to the untreated cells (A-D: Right graphs, ${ }^{*} \mathrm{P}<0.05,{ }^{* *} \mathrm{P}<0.01$ ). DHT, dihydrotestosterone; AR, androgen receptor; PSA, prostate-specific antigen.

factor binding sites in the EPHA3 core promoter region were inferred, one of which was an androgen-responsive element (ARE) for AR binding located between -615 and $-601 \mathrm{bp}$, and the other one ranging from -295 to -261 bp for Spl binding, as shown in Fig. 4A. Based on this, different truncated promoters were used for construction of luciferase reporter plasmids to analyze the EPHA3 promoter activity (Fig. 4B). The results in Fig. $4 \mathrm{C}$ showed that, in $22 \mathrm{Rv} 1$ cells, the promoter luciferase activity of the EPHA3-Luc-789 reporter plasmid with both AR and SP1 binding sites was similarly as high as that of the EPHA3-Luc-317 reporter plasmid with the SP1 binding site only. In addition, AR overexpression also remarkably 
A

$\operatorname{DHT}(\mathrm{nM})$

B

B $\operatorname{Mib}(\mathrm{nM})$

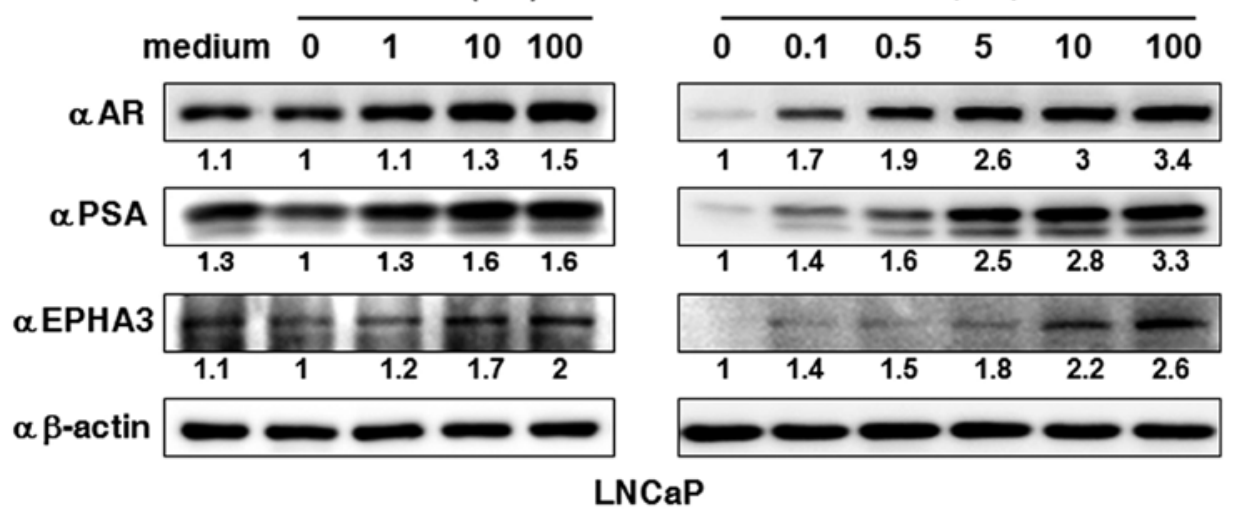

Figure 2. Dose effects of androgen treatment on EPHA3 expression in prostate cancer LNCaP cells. (A) After androgen starvation, LNCaP cells were treated with DHT at final concentrations of 1,10 and $100 \mathrm{nM}$ for $24 \mathrm{~h}$, or (B) Mib at concentrations of 0, 0.1, 0.5, 5, 10 and $100 \mathrm{nM}$. Western blotting was performed to determine protein expression for AR, PSA and EPHA3 levels as described in the text, and $\beta$-actin levels were used as loading controls. Images were analyzed by densitometry for relative protein expression of AR, PSA and EPHA3. The results are shown as relative fold-change, normalized to $\beta$-actin and relative to untreated cells. Mib, mibolerone; DHT, dihydrotestosterone; AR, androgen receptor; PSA, prostate-specific antigen.

A

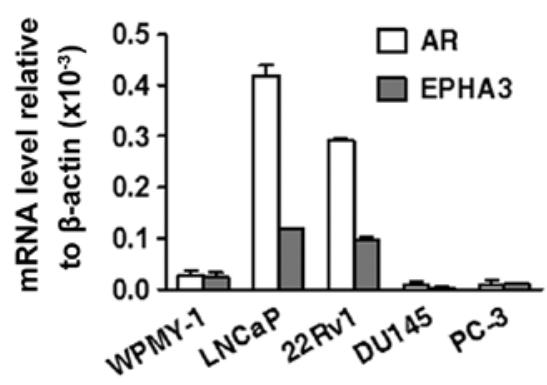

C

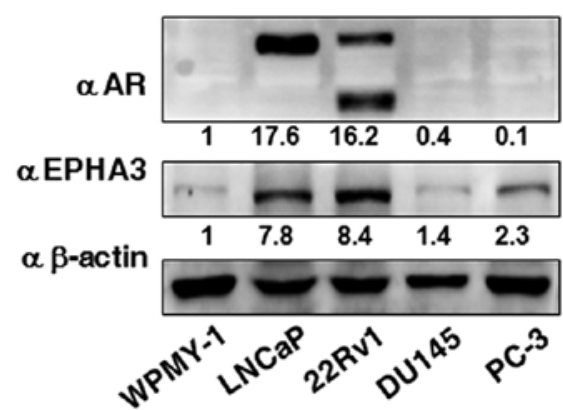

B

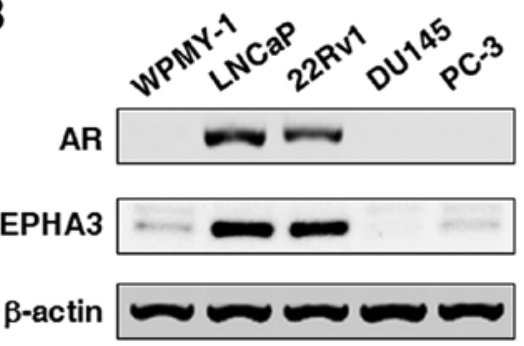

D

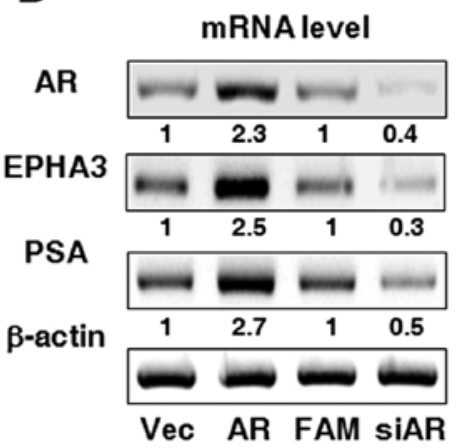

E

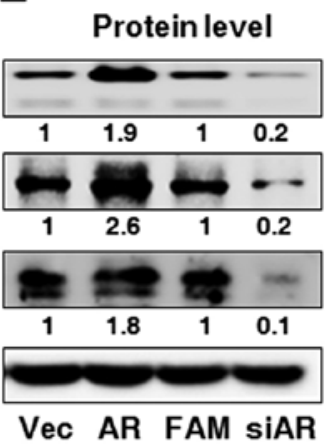

Figure 3. EPHA3 expression is associated with AR expression in androgen-dependent prostate cancer cell lines. (A) EPHA3 and AR mRNA expression in WPMY-1, LNCaP, 22Rv1, DU145 and PC-3 cells was determined by qPCR and then (B) applied to agarose gel electrophoresis. (C) EPHA3 and AR protein expression in these cells was measured by western blotting as described in the text, and $\beta$-actin levels were used as loading controls. The $22 \mathrm{Rv} 1$ cells in 6-well plates were transfected with $3 \mu \mathrm{g}$ of pEGFP-AR (AR) or $50 \mathrm{nM}$ siAR for $48 \mathrm{~h}$ to measure (D) mRNA and (E) protein expression of EPHA3 and AR. The images of agarose gel electrophoresis and western blotting membranes were separately analyzed by densitometry for quantification. The results are shown as the relative fold-change marked below the bands, after being normalized to $\beta$-actin levels and adjusted with conditional control levels. AR, androgen receptor.

increased the EPHA3 promoter activity in both the -789 and -317 truncation plasmids, whereas the siRNA-mediated knockdown of AR markedly decreased the promoter activity in 22Rv1 cells (Fig. 4C). The EPHA3 promoter activity was the lowest in 22Rv1 cells transfected with the EPHA3-Luc-237 plasmid including neither the ARE nor SP1 binding sites, and was not affected by either AR overexpression or siAR interference (Fig. 4C). Compared to the EPHA3-Luc-789 plasmid transfected group, the EPHA3 promoter activity was downregulated by about half in 22Rv1 cells after transfection with the EPHA3-Luc-789 $\triangle$ SP1 plasmid containing modified SP1 binding sites in the GC-rich region, and scarcely influenced by co-transfection with AR or siAR (Fig. 4C).

Transcription factor SP1 still regulates EPHA3 expression even when AR is not exogeneously altered. In order to further 
A
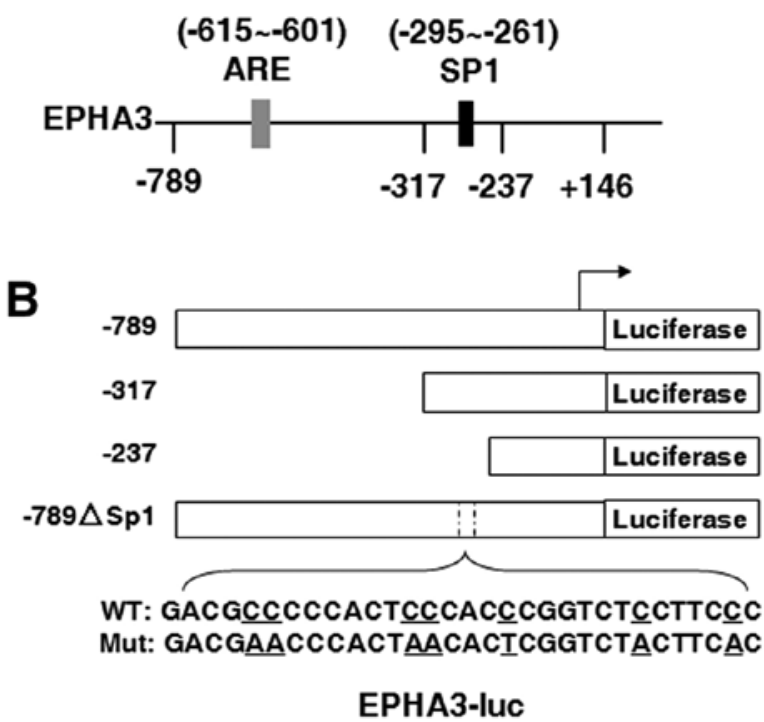

C

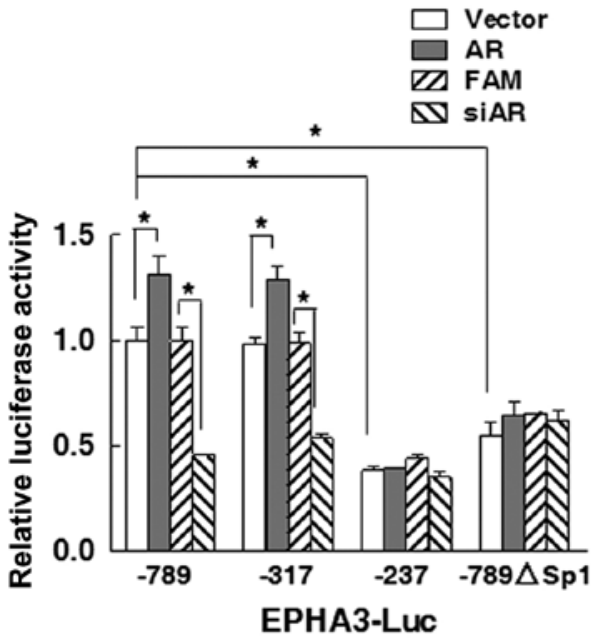

Figure 4. Mutations of the EPHA3 promoter differ in the response to AR signaling in 22Rv1 cells. (A) Schematic representation of the EPHA3 promoter region. Gray box, ARE; black box, SP1 binding sites. (B) EPHA3-Luc plasmids (-789, -317, -237 and -789 $\Delta$ Sp1) were constructed as described in the text. The mutated sequence of the SP1 binding site is shown at the bottom, in which the modified nucleotides are underlined. (C) 22Rv1 cells were co-transfected with various EPHA3-Luc plasmids and pEGFP-AR (AR) or siAR, and internal control pRL-TK for $48 \mathrm{~h}$. Then, luciferase assays were carried out as indicated in the text. The luciferase activity in each group is shown as fold-change normalized to the Renilla luciferase activity and relative to cells treated with EPHA3-Luc-789 plasmid $\left({ }^{*} \mathrm{P}<0.05\right)$. AR, androgen receptor.
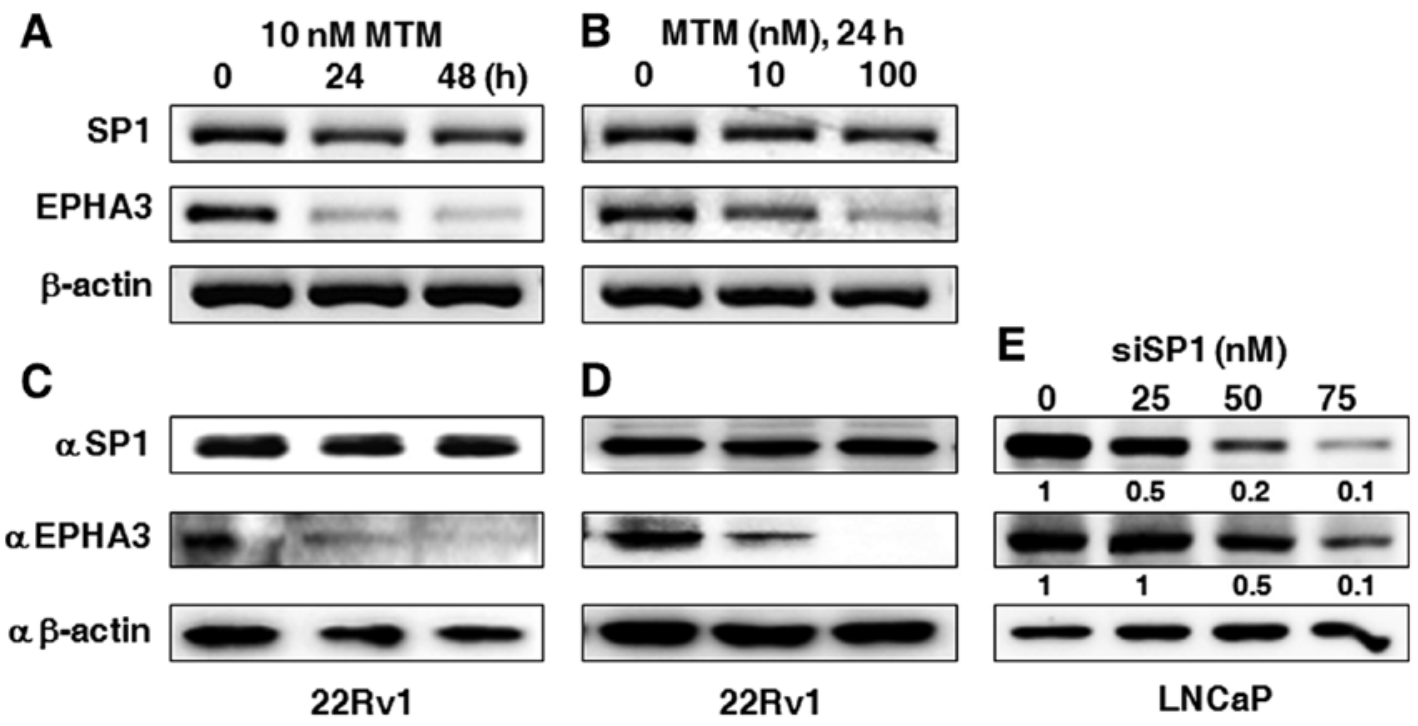

Figure 5. Inhibition of SP1-dependent transcription decreases EPHA3 expression in PCa cells. The 22Rv1 cells were treated with the indicated concentrations of MTM for 24 or $48 \mathrm{~h}$. (A and B) RT-PCR and (C and D) western blotting were performed to measure mRNA and protein expression of SP1 and EPHA3, as described in the text. Additionally, the $\beta$-actin levels were used as loading controls. (E) LNCaP cells were transfected with siSP1 varying from 25 to 75 nM. Western blotting was used to determine protein levels of SP1 and EPHA3, and $\beta$-actin level was used as loading control. The immunoblots were quantified by densitometry, normalized to $\beta$-actin and relative to untreated cells, showing as the relative fold-change value below the bands. PCa, prostate cancer.

characterize the involvement of SP1 in the regulation of EPHA3 expression when AR is not artificially inhibited or overexpressed, MTM, as an inhibitor of SP1 transcription activity, was added to $22 \mathrm{Rv} 1$ cells to determine the effect of SP1 transcriptional activation on EPHA3 expression. The data displayed in Fig. 5A shows that the EPHA3 mRNA level was severely reduced in the presence of $10 \mathrm{nM}$ MTM from 24 to $48 \mathrm{~h}$, or by the treatment with 10 and $100 \mathrm{nM} \mathrm{MTM}$ for $24 \mathrm{~h}$, although the SP1 mRNA level was rarely affected (Fig. 5B).
Accordingly, the EPHA3 protein level was also markedly reduced under the same conditions, while the SP1 protein level was barely changed, as shown in Fig. 5C and D. In addition, specific inhibition of SP1 in LNCaP cells using siSP1 at an increasing gradient mode also gradually abated EPHA3 protein expression (Fig. 5E).

Interaction between $A R$ and SPI contributes to the hormone response of the EPHA3 promoter. Co-IP assay was performed 
A

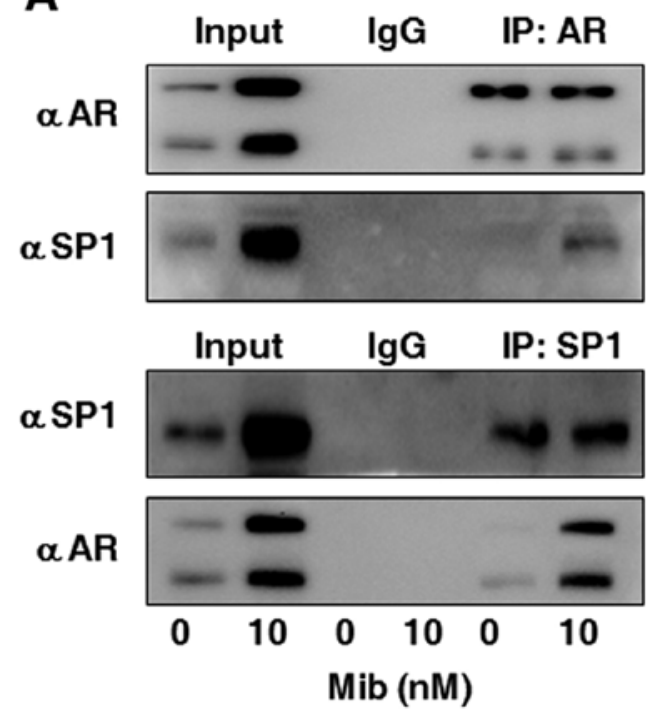

B

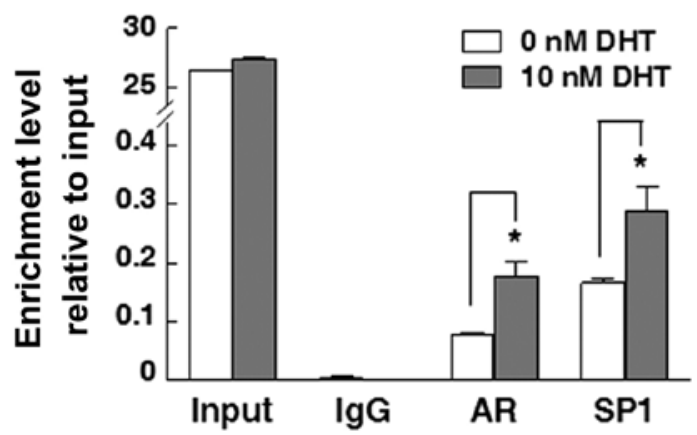

Figure 6. SP1 binds the EPHA3 core promoter. (A) As detailed in the text, using anti-AR antibody, anti-SP1 antibody, and normal rabbit serum IgG as a negative control, the androgen-starved 22Rv1 cells were induced with $10 \mathrm{nM} \mathrm{Mib}$ for $24 \mathrm{~h}$ and harvested for co-IP followed by western blotting, and (B) the androgen-starved 22Rv1 cells were stimulated with $10 \mathrm{nM}$ DHT for ChIP assay followed by RT-qPCR analysis to calculate the target DNA enrichment using the cycle threshold value against input control $\left({ }^{*} \mathrm{P}<0.05\right)$. Mib, mibolerone; DHT, dihydrotestosterone; co-IP, co-immunoprecipitation; ChIP, chromatin IP.

to evaluate whether AR can bind to the Sp1 transcription factor. As shown in Fig. 6A, the AR protein was detected in 22Rv1 cells after immunoprecipitation with antibodies not only to AR but also to SP1 in the presence of Mib. In addition, the SP1 protein was also immunoprecipitated by the SP1 or AR antibody in these cells after Mib stimulation (Fig. 6A). To determine whether androgen-induced EPHA3 expression is regulated through SP1 binding sites in the EPHA3 core promoter region, ChIP assay was carried out to analyze the association of the SP1 protein with specific core promoter regions of EPHA3 in the case of DHT stimulation. As shown in Fig. 6B, both SP1 and AR were found to be bound to the SP1 binding sites in the EPHA3 core promoter region even in the androgen-starvation state. In addition, the SP1 binding capacity was around 2-fold higher than that of AR, and their binding capacities to this GC-rich region were increased almost 2 folds in the presence of DHT.

\section{Discussion}

Although EPHA3 plays an important role in the progression of various invasive and metastatic cancers $(14,15,27,38,39)$, the mechanisms involved in the regulation of EPHA3 are largely unknown. In the current study, we demonstrated that EPHA3 expression was induced by DHT hormone, in PCa cells 22Rv1, at both the mRNA and protein levels in a time- and dose-dependent manner, while the expression of AR and the AR-targeted gene, PSA revealed the increasing trend along with DHT stimulation. Similarly, EPHA3 was also markedly induced in PCa LNCaP cells treated with DHT or Mib. The results indicated that EPHA3 expression is responsive to androgen hormone, which is possibly due to AR regulation since most biological effects of androgens are mediated through the action of nuclear AR as a master regulator of downstream androgen-dependent signaling pathway networks (40). We also found that the EPHA3 mRNA expression level was rather high in the androgen-dependent PCa LNCaP and 22Rv1 cells with higher AR expressions compared with the AR non-expressing prostate stromal cell WPMY-1 and PCa cell lines PC-3 and DU145. This finding is consistent with a previous report that mRNA level of EPHA3 was highly expressed in LNCaP cells (26), and, additionally, so was the EPHA3 protein expression in these cells, i.e., EPHA3 appeared to exhibit a similar expression pattern as the AR at both the mRNA and protein levels. When the AR was overexpressed in 22Rv1 cells, the EPHA3 mRNA and protein levels were significantly increased with the rise of the PSA expression. In contrast, mRNA and protein expressions of PSA and EPHA3 were markedly decreased after knocking down the AR, which clearly suggested that AR activity can affect EPHA3 expression in androgen-dependent PCa cells. A reported study showed that prostate androgen induces the prostate leucine zipper gene promoted EPHA3 expression (27), suggesting from another point of view that the AR is probably associated with EPHA3 regulation.

AR is composed of four domains, the $\mathrm{N}$ terminal transactivation domain, the DNA binding domain, the flexible hinge region and the LBD. Usually, the inactivated AR in the cytoplasm can be translocated into the nucleus upon binding with ligands, such as androgen hormone, and then attached to specific DNA binding sites of ARE loci in the promoter region of the target gene to start the AR-mediated transcription activity (33). In the present study, we assumed that the potential ARE site was between -615 to -601 bp and the SP1 binding sites of highly GC-rich region was between -295 and -261 bp in the EPHA3 promoter region. We found that the SP1 binding sites were indeed required for the AR-mediated EPHA3 promoter activity, while the elimination of the ARE site hardly affected the EPHA3 reporter promoter activity. Specifically, AR regulation of EPHA3 expression 
is not due to direct binding of the AR with the EPHA3 core promoter region, but AR may regulate EPHA3 through a SP1-dependent pathway whereby AR can regulate downstream gene expression not only as a transcription factor binding to ARE sites in the promoter of target genes, but also as an auxiliary transcription factor interacting with the transcription complex.

SP1 is a well-known member of the Sp transcription factor family including SP2, SP3 and SP4, which are implicated in varieties of biological processes (41). SP1 activates gene transcription by binding to specific CG-rich SP-binding sites within gene promoters, and has been considered as a therapeutic target for human cancers, including PCa (41-44). In this study, we demonstrated that EPHA3 expression was markedly downregulated when $22 \mathrm{Rv} 1$ cells were treated with MTM, which inhibited SP1 binding to GC rich promoter region. Similarly, it was also downregulated when LNCaP cells were treated with siSP1 to knock down SP1 expression, which suggested that SP1 may regulate EPHA3 expression as a transcription factor. The co-IP assay further showed that AR forms a complex with SP1. Additionally, the ChIP assay confirmed that SP1 mediated androgen induction of EPHA3 core promoter activity involved with DNA binding. These findings suggested that androgen-initiated AR signaling transduction is achieved through the interaction of AR and SP1 to mediate EPHA3 expression, whereby SP1 is able to bind the EPHA3 core promoter in chromatin responding to AR signaling. This is in accordance with the functional mechanism of AR and the transcription factor SP1 complex to mediate vascular endothelial growth factor (VEGF) expression in PCa cells in response to androgen induction, which is also dependent upon a critical SP1 binding site within the VEGF core promoter (45). Similarly, AR and SP1 induced cyclin dependent kinase inhibitor gene p21 transcription in LNCaP cells through binding to the ARE, as well as SP1 binding site in the p21 promoter after androgen stimulation (46). Our results showed that the binding between SP1 and the EPHA3 core promoter region to induce EPHA3 expression occurred no matter whether androgen exists or not, which may result from constitutively active AR mutants expressed only in $22 \mathrm{Rv} 1$ cells (47-49) and/or incomplete androgen-starvation of $22 \mathrm{Rv} 1$ cells used in our experiments.

Furthermore, it has been previously reported that EPHA3 is significantly increased during the conversion of $\mathrm{LNCaP}$ cells from androgen-dependent (LNCaP-C33) to androgen-independent (LNCaP-C 81) phenotypes using Affymetrix GeneChip array analysis (50). Nevertheless, this does not imply that the increase of EPHA3 expression is independent from androgen induction, or that AR is not associated with EPHA3 regulation in androgen-independent prostate cells as such. Indeed, compared to androgen-dependent $\mathrm{LNCaP}$ cells, the AR level is also increased in androgen-independent LNCaP sublines, and two AR target genes, namely UGT2B15 and UGT2B17, which are not expressed in AR-negative PCa cells, were both positively correlated with upregulated AR in androgen-independent $\mathrm{LNCaP}$ subline, though PSA as one of the AR main targets, was markedly decreased $(51,52)$. This suggested that upregulation of AR targets in androgen-dependent PCa cells may occur in AR positive androgen-independent state. On the other hand, PSA would also be downregulated under androgen-independent condition, e.g., increased microR-100 in androgen-independent PCa cells suppresses PSA expression even if AR transcription activity initiated by ligands is blocked, mostly due to androgen deprivation (53). Other factors, such as the NF- $\mathrm{KB}$ level also mediates PSA expression in androgen-independent PCa cells (54). Thus, there may be additional molecules and other pathways besides AR signaling involved in regulating EPHA3 in AR-positive castration-resistant PCa and various stages of PCa progression, which remains to be further examined.

The present study found that EPHA3 is increased at the transcript and protein expression levels in 22Rv1 cells in a time- and dose-dependent manner under treatment with DHT, and the EPHA3 expression pattern is similar to that of AR in the prostate (cancer) cell lines. AR overexpression or AR inhibition markedly affected the EPHA3 levels, due to the interaction of AR and SP1 as a transcription factor to bind SP1 binding sites in the core promoter region of EPHA3. These findings indicated the association among EPHA3, AR and SP1, which could be useful to gain further insight into the importance of EPHA3 in PCa development and progression and will additionally facilitate our understanding of $\mathrm{AR}$ and $\mathrm{SP} 1$ as targets for the treatment of PCa.

\section{Acknowledgements}

We are grateful to Chuanjun Wen (Nanjing Normal University) for providing us the plasmids.

\section{Funding}

The present study was supported by funding from the National Natural Science Foundation of China (nos. 81172007, 81472415 and 81272850).

\section{Availability of data and materials}

The datasets used during the present study are available from the corresponding author upon reasonable request.

\section{Authors' contributions}

SL, XD, XC and PL conceived and designed the experiments; $\mathrm{XD}, \mathrm{XC}, \mathrm{YP}, \mathrm{YZ}$ and FW performed experiments; SL, XD, XC, PL, YG, XW and SY analyzed the data; PL, YG, XW, SY and SL provided reagents, materials and tools; SL, YG, SY and XW wrote and reviewed the manuscript. All authors read and approved the manuscript and agree to be accountable for all aspects of the research in ensuring that the accuracy or integrity of any part of the work are appropriately investigated and resolved.

\section{Ethics approval and consent to participate}

Not applicable.

\section{Patient consent for publication}

Not applicable. 


\section{Competing interests}

The authors state that they have no competing interests.

\section{References}

1. Hood G, Laufer-Amorim R, Fonseca-Alves CE and Palmieri C: Overexpression of ephrin A3 receptor in canine prostatic carcinoma. J Comp Pathol 154: 180-185, 2016.

2. Xi HQ, Wu XS, Wei B and Chen L: Aberrant expression of EphA3 in gastric carcinoma: Correlation with tumor angiogenesis and survival. J Gastroenterol 47: 785-794, 2012.

3. Nasri B, Inokuchi M, Ishikawa T, Uetake H, Takagi Y, Otsuki S, Kojima K and Kawano T: High expression of EphA3 (erythropoietin-producing hepatocellular A3) in gastric cancer is associated with metastasis and poor survival. BMC Clin Pathol 17: 8, 2017.

4. Tímár J, Mészáros L, Ladányi A, Puskás LG and Rásó E Melanoma genomics reveals signatures of sensitivity to bio- and targeted therapies. Cell Immunol 244: 154-157, 2006.

5. Caivano A, La Rocca F, Laurenzana I, Annese T, Tamma R, Famigliari U, Simeon V, Trino S, De Luca L, Villani O, et al: Epha3 acts as proangiogenic factor in multiple myeloma. Oncotarget 8: 34298-34309, 2017.

6. La Rocca F, Airoldi I, Di Carlo E, Marotta P, Falco G, Simeon V, Laurenzana I, Trino S, De Luca L, Todoerti K, et al: EphA3 targeting reduces in vitro adhesion and invasion and in vivo growth and angiogenesis of multiple myeloma cells. Cell Oncol 40: 483-496, 2017.

7. Lu CY, Yang ZX, Zhou L, Huang ZZ, Zhang HT, Li J, Tao KS and Xie BZ: High levels of EphA3 expression are associated with high invasive capacity and poor overall survival in hepatocellular carcinoma. Oncol Rep 30: 2179-2186, 2013.

8. Day BW, Stringer BW, Al-Ejeh F, Ting MJ, Wilson J, Ensbey KS, Jamieson PR, Bruce ZC, Lim YC, Offenhäuser C, et al: EphA3 maintains tumorigenicity and is a therapeutic target in glioblastoma multiforme. Cancer Cell 23: 238-248, 2013.

9. Li M, Yang C, Liu X, Yuan L, Zhang F, Wang M, Miao D, Gu X, Jiang S, Cui B, et al: EphA3 promotes malignant transformation of colorectal epithelial cells by upregulating oncogenic pathways. Cancer Lett 383: 195-203, 2016.

10. Brantley DM, Cheng N, Thompson EJ, Lin Q, Brekken RA, Thorpe PE, Muraoka RS, Cerretti DP, Pozzi A, Jackson D, et al: Soluble Eph A receptors inhibit tumor angiogenesis and progression in vivo. Oncogene 21: 7011-7026, 2002.

11. Swords RT, Greenberg PL, Wei AH, Durrant S, Advani AS Hertzberg MS, Jonas BA, Lewis ID, Rivera G, Gratzinger D, et al: $\mathrm{KB} 004$, a first in class monoclonal antibody targeting the receptor tyrosine kinase EphA3, in patients with advanced hematologic malignancies: Results from a phase 1 study. Leuk Res 50: 123-131, 2016.

12. Ferluga S, Tomé CM, Herpai DM, D'Agostino R and Debinski W: Simultaneous targeting of Eph receptors in glioblastoma. Oncotarget 7: 59860-59876, 2016.

13. Charmsaz S, Al-Ejeh F, Yeadon TM, Miller KJ, Smith FM, Stringer BW, Moore AS, Lee FT, Cooper LT, Stylianou C, et al EphA3 as a target for antibody immunotherapy in acute lymphoblastic leukemia. Leukemia 31: 1779-1787, 2017.

14. Janes PW, Slape CI, Farnsworth RH, Atapattu L, Scott AM and Vail ME: EphA3 biology and cancer. Growth Factors 32: 176-189, 2014.

15. Lodola A, Giorgio C, Incerti M, Zanotti I and Tognolini M: Targeting Eph/ephrin system in cancer therapy. Eur J Med Chem 142: 152-162, 2017.

16. Herath NI, Spanevello MD, Doecke JD, Smith FM, Pouponnot C and Boyd AW: Complex expression patterns of Eph receptor tyrosine kinases and their ephrin ligands in colorectal carcinogenesis. Eur J Cancer 48: 753-762, 2012.

17. $\mathrm{Xi} H Q$ and Zhao P: Clinicopathological significance and prognostic value of EphA3 and CD133 expression in colorectal carcinoma. J Clin Pathol 64: 498-503, 2011.

18. Andretta E, Cartón-García F, Martínez-Barriocanal Á, de Marcondes PG, Jimenez-Flores LM, Macaya I, Bazzocco S, Bilic J, Rodrigues P, Nieto R, et al: Investigation of the role of tyrosine kinase receptor EPHA3 in colorectal cancer. Sci Rep 7: 41576, 2017.

19. Vecchi M, Confalonieri S, Nuciforo P, Viganò MA, Capra M, Bianchi M, Nicosia D, Bianchi F, Galimberti V, Viale G, et al: Breast cancer metastases are molecularly distinct from their primary tumors. Oncogene 27: 2148-2158, 2008.
20. Clifford N, Smith LM, Powell J, Gattenlöhner S, Marx A and O'Connor R: The EphA3 receptor is expressed in a subset of rhabdomyosarcoma cell lines and suppresses cell adhesion and migration. J Cell Biochem 105: 1250-1259, 2008.

21. Chiappalupi S, Riuzzi F, Fulle S, Donato R and Sorci G: Defective RAGE activity in embryonal rhabdomyosarcoma cells results in high PAX7 levels that sustain migration and invasiveness. Carcinogenesis 35: 2382-2392, 2014.

22. Zhuang G, Song W, Amato K, Hwang Y, Lee K, Boothby M, Ye F, Guo Y, Shyr Y, Lin L, et al: Effects of cancer-associated EPHA3 mutations on lung cancer. J Natl Cancer Inst 104: 1182-1197, 2012

23. Peng J, Wang Q, Liu H, Ye M, Wu X and Guo L: EPHA3 regulates the multidrug resistance of small cell lung cancer via the PI3K/BMX/STAT3 signaling pathway. Tumour Biol 37: 11959-11971, 2016

24. Hafner C, Schmitz G, Meyer S, Bataille F, Hau P, Langmann T, Dietmaier W, Landthaler M and Vogt T: Differential gene expression of Eph receptors and ephrins in benign human tissues and cancers. Clin Chem 50: 490-499, 2004.

25. Vail ME, Murone C, Tan A, Hii L, Abebe D, Janes PW, Lee FT, Baer M, Palath V, Bebbington C, et al: Targeting EphA3 inhibits cancer growth by disrupting the tumor stromal microenvironment. Cancer Res 74: 4470-4481, 2014

26. Fox BP, Tabone CJ and Kandpal RP: Potential clinical relevance of Eph receptors and ephrin ligands expressed in prostate carcinoma cell lines. Biochem Biophys Res Commun 342: 1263-1272, 2006.

27. Wu R, Wang H, Wang J, Wang P, Huang F, Xie B, Zhao Y, Li S and Zhou J: EphA3, induced by PC-1/PrLZ, contributes to the malignant progression of prostate cancer. Oncol Rep 32: 2657-2665, 2014

28. World Health Organization: Global battle against cancer won't be won with treatment alone-effective prevention measures urgently needed to prevent cancer crisis. Cent Eur J Public Health 22: 23, 28, 2014.

29. Roviello G, Sigala S, Sandhu S, Bonetta A, Cappelletti MR, Zanotti L, Bottini A, Sternberg CN, Fox SB and Generali D: Role of the novel generation of androgen receptor pathway targeted agents in the management of castration-resistant prostate cancer: A literature based meta-analysis of randomized trials. Eur J Cancer 61: 111-121, 2016.

30. Komura K, Jeong SH, Hinohara K, Qu F, Wang X, Hiraki M, Azuma H, Lee GS, Kantoff PW and Sweeney CJ: Resistance to docetaxel in prostate cancer is associated with androgen receptor activation and loss of KDM5D expression. Proc Natl Acad Sci USA 113: 6259-6264, 2016.

31. Geynisman DM,Plimack ER and Zibelman M: Second-generation androgen receptor-targeted therapies in nonmetastatic castration-resistant prostate cancer: Effective early intervention or intervening too early? Eur Urol 70: 971-973, 2016.

32. Juang HH, Hsieh ML and Tsui KH: Testosterone modulates mitochondrial aconitase in the full-length human androgen receptor-transfected PC-3 prostatic carcinoma cells. J Mol Endocrinol 33: 121-132, 2004.

33. Zhao H, Zhu C, Qin C, Tao T, Li J, Cheng G, Li P, Cao Q, Meng X, Ju X, et al: Fenofibrate down-regulates the expressions of androgen receptor (AR) and AR target genes and induces oxidative stress in the prostate cancer cell line LNCaP. Biochem Biophys Res Commun 432: 320-325, 2013.

34. Lee WS, Kwon J, Yun DH, Lee YN, Woo EY, Park MJ, Lee JS, Han YH and Bae IH: Specificity protein 1 expression contributes to Bcl-w-induced aggressiveness in glioblastoma multiforme. Mol Cells 37: 17-23, 2014.

35. Simon P: Q-Gene: Processing quantitative real-time RT-PCR data. Bioinformatics 19: 1439-1440, 2003.

36. Dottori M, Down M, Hüttmann A, Fitzpatrick DR and Boyd AW: Cloning and characterization of EphA3 (Hek) gene promoter: DNA methylation regulates expression in hematopoietic tumor cells. Blood 94: 2477-2486, 1999

37. Liao X, Tang S, Thrasher JB, Griebling TL and Li B: Small-interfering RNA-induced androgen receptor silencing leads to apoptotic cell death in prostate cancer. Mol Cancer Ther 4: 505-515, 2005.

38. Boyd AW, Ward LD, Wicks IP, Simpson RJ, Salvaris E, Wilks A, Welch K, Loudovaris M, Rockman S and Busmanis I: Isolation and characterization of a novel receptor-type protein tyrosine kinase (hek) from a human pre-B cell line. J Biol Chem 267: 3262-3267, 1992. 
39. Pasquale EB: Eph receptors and ephrins in cancer: Bidirectional signalling and beyond. Nat Rev Cancer 10: 165-180, 2010.

40. Matsumoto T, Sakari M, Okada M, Yokoyama A, Takahashi S, Kouzmenko A and Kato S: The androgen receptor in health and disease. Annu Rev Physiol 75: 201-224, 2013.

41. Vizcaíno C, Mansilla S and Portugal J: Sp1 transcription factor: A long-standing target in cancer chemotherapy. Pharmacol Ther 152: 111-124, 2015.

42. Beishline K and Azizkhan-Clifford J: Sp1 and the 'hallmarks of cancer'. FEBS J 282: 224-258, 2015.

43. Safe S, Imanirad P, Sreevalsan S, Nair V and Jutooru I: Transcription factor $\mathrm{Sp} 1$, also known as specificity protein 1 as a therapeutic target. Expert Opin Ther Targets 18: 759-769, 2014.

44. Sankpal UT, Goodison S, Abdelrahim M and Basha R: Targeting Sp1 transcription factors in prostate cancer therapy. Med Chem 7: 518-525, 2011.

45. Eisermann K, Broderick CJ, Bazarov A, Moazam MM and Fraizer GC: Androgen up-regulates vascular endothelial growth factor expression in prostate cancer cells via an Sp1 binding site. Mol Cancer 12: 7, 2013.

46. Lu S, Jenster G and Epner DE: Androgen induction of cyclin-dependent kinase inhibitor p21 gene: Role of androgen receptor and transcription factor Spl complex. Mol Endocrinol 14: 753-760, 2000

47. Tepper CG, Boucher DL, Ryan PE, Ma AH, Xia L, Lee LF, Pretlow TG and Kung HJ: Characterization of a novel androgen receptor mutation in a relapsed CWR22 prostate cancer xenograft and cell line. Cancer Res 62: 6606-6614, 2002.
48. Attardi BJ, Burgenson J, Hild SA and Reel JR: Steroid hormonal regulation of growth, prostate specific antigen secretion, and transcription mediated by the mutated androgen receptor in CWR22Rv1 human prostate carcinoma cells. Mol Cell Endocrinol 222: 121-132, 2004.

49. Li Y, Hwang TH, Oseth LA, Hauge A, Vessella RL, Schmechel SC, Hirsch B, Beckman KB, Silverstein KA and Dehm SM: AR intragenic deletions linked to androgen receptor splice variant expression and activity in models of prostate cancer progression. Oncogene 31: 4759-4767, 2012.

50. Singh AP, Bafna S, Chaudhary K, Venkatraman G, Smith L, Eudy JD, Johansson SL, Lin MF and Batra SK: Genome-wide expression profiling reveals transcriptomic variation and perturbed gene networks in androgen-dependent and androgen-independent prostate cancer cells. Cancer Lett 259: 28-38, 2008.

51. Yuan TC, Veeramani S and Lin MF: Neuroendocrine-like prostate cancer cells: Neuroendocrine transdifferentiation of prostate adenocarcinoma cells. Endocr Relat Cancer 14: 531-547, 2007.

52. Bao BY, Chuang BF, Wang Q, Sartor O, Balk SP, Brown M, Kantoff PW and Lee GS: Androgen receptor mediates the expression of UDP-glucuronosyltransferase 2 B15 and B17 genes. Prostate 68: 839-848, 2008.

53. Wang Y, Wang Y, Liu Q, Xu G, Mao F, Qin T, Teng H, Cai W, Yu P, Cai T, et al: Comparative RNA-seq analysis reveals potential mechanisms mediating the conversion to androgen independence in an LNCaP progression cell model. Cancer Lett 342: 130-138, 2014.

54. Chen CD and Sawyers CL: NF-kappa B activates prostate-specific antigen expression and is upregulated in androgen-independent prostate cancer. Mol Cell Biol 22: 2862-2870, 2002. 